\title{
Evidence of significant energy input in the late phase of a solar flare from NuSTAR $x-$ ray observations
}

Kuhar, Matej; Krucker, Sam; Hannah, lain G.; Glesener, Lindsay; Saint-Hilaire, Pascal; Grefenstette, Brian W.; Hudson, Hugh S.; White, Stephen M.; Smith, David M.; Marsh, Andrew J.

Total number of authors:

18

Published in:

Astrophysical Journal

Link to article, DOI:

$10.3847 / 1538-4357 / 835 / 1 / 6$

Publication date:

2017

Document Version

Publisher's PDF, also known as Version of record

Link back to DTU Orbit

Citation $(A P A)$ :

Kuhar, M., Krucker, S., Hannah, I. G., Glesener, L., Saint-Hilaire, P., Grefenstette, B. W., Hudson, H. S., White, S. M., Smith, D. M., Marsh, A. J., Wright, P. J., Boggs, S. E., Christensen, F. E., Craig, W. W., Hailey, C. J., Harrison, F. A., Stern, D., \& Zhang, W. W. (2017). Evidence of significant energy input in the late phase of a solar flare from NuSTAR x-ray observations. Astrophysical Journal, 835(1). https://doi.org/10.3847/1538$4357 / 835 / 1 / 6$

\section{General rights}

Copyright and moral rights for the publications made accessible in the public portal are retained by the authors and/or other copyright owners and it is a condition of accessing publications that users recognise and abide by the legal requirements associated with these rights.

- Users may download and print one copy of any publication from the public portal for the purpose of private study or research.

- You may not further distribute the material or use it for any profit-making activity or commercial gain

- You may freely distribute the URL identifying the publication in the public portal 


\title{
EVIDENCE OF SIGNIFICANT ENERGY INPUT IN THE LATE PHASE OF A SOLAR FLARE FROM NUSTAR X-RAY OBSERVATIONS
}

\author{
Matej Kuhar $^{1,2}$, Säm Krucker ${ }^{1,3}$, Iain G. Hannah ${ }^{4}$, Lindsay Glesener ${ }^{5}$, Pascal Saint-Hilaire ${ }^{3}$, \\ Brian W. Grefenstette ${ }^{6}$, Hugh S. Hudson ${ }^{3,7}$, Stephen M. White ${ }^{8}$, David M. Smith ${ }^{9}$, Andrew J. Marsh', \\ Paul J. Wright ${ }^{4}$, Steven E. Boggs ${ }^{3}$, Finn E. Christensen ${ }^{10}$, William W. Craig ${ }^{3,11}$, Charles J. Hailey ${ }^{12}$, \\ Fiona A. Harrison ${ }^{6}$, Daniel Stern ${ }^{13}$, and William W. Zhang ${ }^{14}$ \\ ${ }^{1}$ University of Applied Sciences and Arts Northwestern Switzerland, Bahnhofstrasse 6, 5210 Windisch, Switzerland \\ ${ }^{2}$ Institute for Particle Physics, ETH Zürich, 8093 Zürich, Switzerland \\ ${ }^{3}$ Space Sciences Laboratory, University of California, Berkeley, CA 94720-7450, USA \\ ${ }^{4}$ SUPA School of Physics \& Astronomy, University of Glasgow, Glasgow G12 8QQ, UK \\ ${ }^{5}$ School of Physics and Astronomy, University of Minnesota-Twin Cities, Minneapolis, MN 55455, USA \\ ${ }^{6}$ Cahill Center for Astrophysics, 1216 E. California Boulevard, California Institute of Technology, Pasadena, CA 91125, USA \\ ${ }^{7}$ School of Physics and Astronomy, University of Glasgow, Glasgow G12 8QQ, UK \\ ${ }^{8}$ Air Force Research Laboratory, Albuquerque, NM, USA \\ ${ }^{9}$ Physics Department and Santa Cruz Institute for Particle Physics, University of California, Santa Cruz, 1156 High Street, Santa Cruz, CA 95064, USA \\ ${ }^{10}$ DTU Space, National Space Institute, Technical University of Denmark, Elektrovej 327, DK-2800 Lyngby, Denmark \\ ${ }^{11}$ Lawrence Livermore National Laboratory, Livermore, CA 94550, USA
${ }^{12}$ Columbia Astrophysics Laboratory, Columbia University, New York, NY 10027, USA \\ ${ }^{13}$ Jet Propulsion Laboratory, California Institute of Technology, 4800 Oak Grove Drive, Pasadena, CA 91109, USA \\ ${ }^{14}$ NASA Goddard Space Flight Center, Greenbelt, MD 20771, USA \\ Received 2016 October 20; revised 2016 November 22; accepted 2016 November 30; published 2017 January 16
}

\begin{abstract}
We present observations of the occulted active region AR 12222 during the third Nuclear Spectroscopic Telescope ARray (NuSTAR) solar campaign on 2014 December 11, with concurrent Solar Dynamics Observatory (SDO)/ AIA and FOXSI-2 sounding rocket observations. The active region produced a medium-size solar flare 1 day before the observations, at $\sim 18$ UT on 2014 December 10, with the post-flare loops still visible at the time of NuSTAR observations. The time evolution of the source emission in the SDO/AIA $335 \AA$ channel reveals the characteristics of an extreme-ultraviolet late-phase event, caused by the continuous formation of new post-flare loops that arch higher and higher in the solar corona. The spectral fitting of NuSTAR observations yields an isothermal source, with temperature 3.8-4.6 MK, emission measure $(0.3-1.8) \times 10^{46} \mathrm{~cm}^{-3}$, and density estimated at $(2.5-6.0) \times 10^{8} \mathrm{~cm}^{-3}$. The observed AIA fluxes are consistent with the derived NuSTAR temperature range, favoring temperature values in the range of 4.0-4.3 MK. By examining the post-flare loops' cooling times and energy content, we estimate that at least 12 sets of post-flare loops were formed and subsequently cooled between the onset of the flare and NUSTAR observations, with their total thermal energy content an order of magnitude larger than the energy content at flare peak time. This indicates that the standard approach of using only the flare peak time to derive the total thermal energy content of a flare can lead to a large underestimation of its value.
\end{abstract}

Key words: Sun: flares - Sun: particle emission - Sun: X-rays, gamma rays

\section{INTRODUCTION}

The Nuclear Spectroscopic Telescope ARray (NuSTAR) is a focusing hard X-ray (HXR) telescope operating in the energy range from 3 to $79 \mathrm{keV}$ (Harrison et al. 2013). While primarily designed to observe far, faint astrophysical sources such as active galactic nuclei, black holes, and supernova remnants, it is also capable of observing the Sun. With its focusing optics system, it can directly observe HXRs from previously undetected sources on the Sun owing to its 10 times higher effective area and orders-of-magnitude-reduced background when compared to state-of-the-art solar HXR instruments such as RHESSI (Lin et al. 2002). However, because it is optimized for observations of astrophyscial objects, NuSTAR experiences some technical challenges when observing the Sun; these include ghost-rays and low throughput. Ghost-rays are unfocused, single-bounced photons (in contrast to properly focused photons that reflect twice off the Wolter-I mirrors) coming from sources outside the field of view (FOV; Madsen et al. 2015). The throughput of NuSTAR's focal plane detector electronics, with a maximum of 400 counts per second per telescope, can effectively diminish the hard X-ray sensitivity in the presence of extremely bright sources (Grefenstette et al. 2016), making detections of fainter spectral components (such as a nonthermal component) difficult.

Despite these challenges, NUSTAR has begun to provide critical new observations of faint X-ray sources on the Sun (Hannah et al. 2016), giving us new insights into the coronal heating problem and particle energization in solar flares. In that respect, occulted active regions are priority targets in the planning of NuSTAR observations. With the brightest emission from the footpoints and low corona hidden, NuSTAR can search for a faint coronal signature of heated material and particle acceleration. In order to maximize $N U S T A R$ livetime and minimize ghost-rays during these observations, they should be carried out during low-activity periods (preferably with no other active sources on the disk).

In this paper, we analyze the occulted active region $\mathrm{AR}$ 12222, which produced a C5.9 GOES (Geostationary Operational Environmental Satellite) class flare $\sim 24 \mathrm{hr}$ before NuSTAR observations. AR 12222 was observed in the third NuSTAR solar campaign on 2014 December 11. The active region was also observed by the Solar TErrestrial RElations 
Observatory (STEREO), the Atmospheric Imaging Assembly on Solar Dynamics Observatory (SDO/AIA), and the second launch of the Focusing Optics X-ray Solar Imager (FOXSI-2) sounding rocket. The goal of this paper is to analyze the time evolution of the X-ray and extreme-ultraviolet (EUV) emission of the observed source above the solar limb in the context of the flare evolution scenario proposed by Woods et al. (2011) and Woods (2014). In these papers, the authors argue that flares may have four distinct phases in their evolution: (1) impulsive phase (best seen in HXRs), (2) gradual phase seen in soft X-ray (SXR)/EUV from the post-flare loops, (3) coronal dimming, best seen in the $171 \AA$ line, and (4) an EUV late phase, best seen as a second peak in the $335 \AA$ line a few hours (up to 6) after the flare onset. The explanation of the EUV late-phase emission lies in the formation of subsequent flare loops, overlying the original flare loops, which result from the reconnection of magnetic fields higher than those that reconnected during the flare's impulsive phase. Similar observations of "giant post-flare loops" and "giant arches" can be found in MacCombie \& Rust (1979), Svestka et al. (1982), Švestka (1984), Švestka et al. (1995), Fárník et al. (1996), Parenti et al. (2010), and West \& Seaton (2015), among others; a theoretical model of the subsequent magnetic reconnections (and its successful description of the flare SOL1973-07-29T13) is given in Kopp \& Poletto (1984). More recently, Liu et al. (2013) proposed that the subsequent loop system(s) is produced by magnetic reconnection of the overlying active region magnetic field lines and the loop arcade produced by the flare, adding more complexity to the theoretical description of these events.

This paper is structured as follows. In Section 2 we give an overview of NUSTAR, SDO/AIA, STEREO, and FOXSI-2 observations of AR 12222. We present the results of NuSTAR spectroscopy in Section 3, along with the comparison of NuSTAR-derived parameters with observations in other wavelengths. The discussion of the results, as well as possible future studies, is presented in Section 4.

\section{OBSERVATIONS}

The data presented in this paper come from the third set of solar observations with NuSTAR, which were carried out on 2014 December 11. The observations consisted of observations of the north pole region (quiet-Sun observations) and the solar limb (from 18:39:00 to 19:04:00 UT) that is discussed in this paper.

The target for the limb pointing and of this study is the active region AR 12222, located $\sim 35^{\circ}$ behind the south-west solar limb at the time of the NuSTAR observations. AR 12222 produced a GOES C5.9 flare 1 day before the NUSTAR observations, at 18 UT on 2014 December 10. Figure 1 presents the time evolution of the GOES flux, the seven SDO/ AIA EUV channels, and the AIA-derived Fe XVI and Fe XVIII fluxes from flare onset until more than a day later. The NuSTAR observing period is indicated with vertical dashed lines. Smaller spikes in the GOES curve between the flare and NUSTAR observations represent various fainter flares coming from other active regions (AR 12233, AR 12230, AR 12235) on the solar disk. Due to the high occultation, the estimate of the GOES class as given above of the flare SOL2014-12-10T18 is a severe lower limit of the actual GOES class. The STEREO satellites can generally be used to give a prediction of the actual GOES class as they view the Sun from a different angle (Nitta et al. 2013). Even though STEREO-A was at the right location at an angle of $\sim 175^{\circ}$ with respect to Earth, it was not observing during the main and gradual phases of the flare; therefore, we cannot give an accurate GOES class estimate for this flare.

The time evolution of fluxes in different AIA channels reveals two main characteristics of an EUV late-phase event, as described in Woods et al. (2011) and Woods (2014): a second (in this case weaker) peak in the $335 \AA$ line a few hours after the flare, and coronal dimming in the $171 \AA$ line with the local minimum $\sim 5 \mathrm{hr}$ after the flare. As previously noted (e.g., Stewart et al. 1974; Rust \& Hildner 1976; Hudson et al. 1998; Zarro et al. 1999; Howard \& Harrison 2004; McIntosh et al. 2007), there is a strong correlation between coronal dimming and coronal mass ejection (CME) events; indeed, a strong CME with the velocity of $\sim 1000 \mathrm{~km} \mathrm{~s}^{-1}$ was associated with this nominally C-class flare. ${ }^{15}$

The olive curve in Figure 1 presents the time evolution of the Fe XVIII line flux. An estimate of the emission in the Fe XVIII line can be constructed from the $94 \AA$ line, by subtracting the lower-temperature responses from the $171 \AA, 193 \AA$, and/or $211 \AA$ channels (see Reale et al. 2011; Testa \& Reale 2012; Warren et al. 2012; Del Zanna 2013). In obtaining the Fe XVIII flux, we followed the approach of Del Zanna (2013), using the formula

$$
\begin{aligned}
F(\mathrm{Fe} \text { XVIII }) \approx & F(94 \AA) \\
& -F(211 \AA) / 120-F(171 \AA) / 450,
\end{aligned}
$$

where $F(\mathrm{Fe}$ XVIII) is the Fe XVIII flux, and $F(94 \AA), F(211 \AA)$, and $F(171 \AA)$ are the fluxes in the 94,211 , and $171 \AA$ channels, respectively. The Fe XVIII line has a strong response in the temperature range from $\sim 3$ to $\sim 10 \mathrm{MK}$, with the peak around 6.5 MK. The Fe XVIII time evolution shows a strong peak due to the flare, with a long decay phase lasting past the NuSTAR observations.

Similar to the Fe XVIII line, a lower-temperature Fe XVI line can be constructed from the 335 and $171 \AA$ lines (Del Zanna 2013):

$$
F(\mathrm{Fe} \mathrm{XVI}) \approx F(335 \AA)-F(171 \AA) / 70 .
$$

Similar to Fe XVIII, the above formula is just an approximation of the Fe XVI flux. The Fe XVI line has a temperature response of similar shape to the Fe XVIII line, with its peak at a lower temperature of $\sim 2.5 \mathrm{MK}$. The time evolution of the Fe XVI flux is also shown in Figure 1. It is characterized by a strong dip followed by the initial rise, soon after which a decrease is observed, due to the fact that the flare becomes weaker. After $\sim 8$ UT on 2014 December 11, the time evolution of Fe XVI flux is determined by fore- and background emission along the line of sight, making the post-flare loops no longer observable in this line.

The evolution of 5-minute integrated NuSTAR fluxes (blue dots) and Fe XVIII fluxes (olive line) is given in the inset of Figure 1. The NuSTAR and Fe XVIII time evolutions show similar behavior, with the (slow) decay rate of the two agreeing within the error bars and the only difference being the steeper decay of NUSTAR flux toward the end of the observation, which is likely an instrumental effect. The NUSTAR focal plane

\footnotetext{
15 Data taken from the LASCO CME Catalog: http://cdaw.gsfc.nasa.gov/ CME_list/.
} 


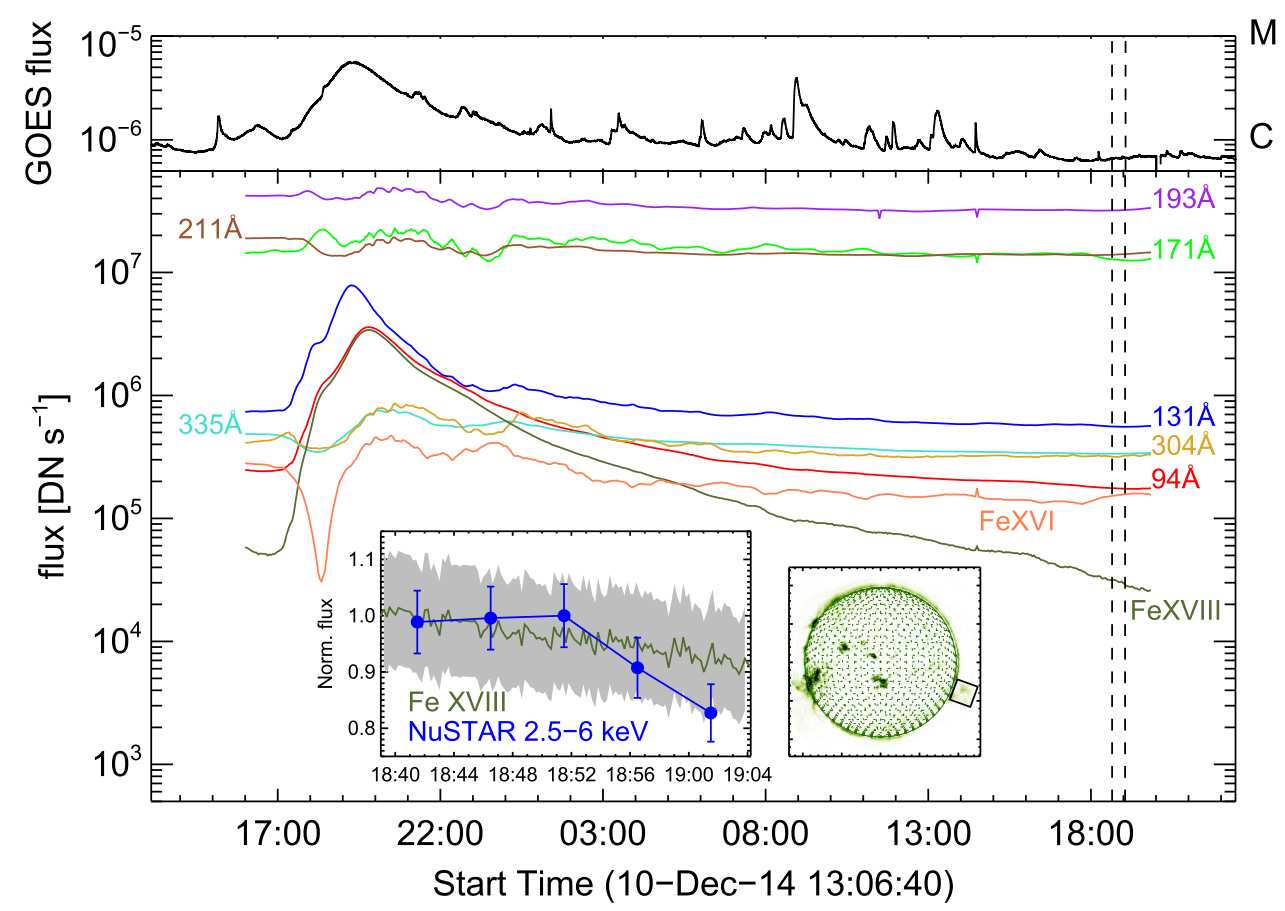

Figure 1. Time profiles of GOES, seven EUV channels of SDO/AIA, Fe XVI, Fe XVIII, and NuSTAR FPMB fluxes from the flaring area above the west limb as marked by the black box in the Fe XVIII map in the inset. Vertical dashed lines represent the time range of NuSTAR observations of the occulted active region AR 12222. The inner plot shows the normalized Fe XVIII (olive line) and NuSTAR fluxes (blue dots) during the observations. The gray shaded area represents an assumed uncertainty of $10 \%$ in the Fe XVIII flux.

consists of a $2 \times 2$ array of CdZnTe detectors, which are divided into quadrants by a chip gap (Harrison et al. 2013). As the telescope pointing drifted slowly during the observations, the gap covered part of the area used for calculating the flux. Therefore, it is probable that the steeper decay of the NUSTAR emission toward the end of the observation is not due to solar variability, but rather a consequence of the telescope drift. This might also have some effect on the determination of the temperature and emission measure of the source, which will be discussed in the following sections.

Due to the slow decay of Fe XVIII emission, we were able to make Fe XVIII images even at the time of NuSTAR observations 1 day after the flare onset (see Figure 2). The upper row presents the Fe XVIII maps of the flare onset, the post-flare loops $6 \mathrm{hr}$ after the flare, and the remaining features $20 \mathrm{hr}$ after the flare. Left and middle panels in the bottom row present 25minute integrated NuSTAR images above $2 \mathrm{keV}$ from focal plane modules A (FPMA) and B (FPMB). Dashed lines denote the area covered by the gap during the observations that is further enlarged due to the drift of the telescope. As the drift was dominantly along the $x$-direction ( $45 "$ in total) and negligible in the $y$-direction, the area affected by the gap is much larger in the $x$-direction. The region of interest for the analysis that will be presented in the next section, with an area of $50^{\prime \prime} \times 50^{\prime \prime}=2500^{\prime \prime 2}$, is marked by the white box. The last image in the bottom right corner is the 25-minute integrated (same time range as NuSTAR) Fe XVIII map of the source, together with the $30 \%, 50 \%, 70 \%$, and $90 \%$ contours of NUSTAR emission in blue. As the uncertainty in NuSTAR absolute pointing accuracy is relatively large (see Grefenstette et al. 2016; Hannah et al. 2016), the NuSTAR image was shifted by $-100^{\prime \prime}$ and $25^{\prime \prime}$ in the $x$ and $y$ directions, respectively, in order to match the Fe XVIII source location. NUSTAR and Fe XVIII maps show the same sources, such as the top parts of the coronal loops, and the high emission source above them (MacCombie \& Rust 1979).

In Figure 3 we present the STEREO-A image of active region AR 12222 an hour before the NuSTAR observation. The orange line shows the solar limb as viewed from Earth, while the red line is a projection of the line of sight from Earth to the NuSTAR source, passing right above AR 12222 located at $\sim\left[-730^{\prime \prime},-330^{\prime \prime}\right]$ in the STEREO-A $195 \AA$ image. The NUSTAR source is not evident in this image as the $195 \AA$ channel is sensitive only to lower temperatures. From STEREO images, it is possible to calculate the height of the post-flare loops, defined as the distance between AR 12222 and the midpoint of the line that minimizes the distance between the Earth-Sun line of sight and the radial extension above the active region. We estimate this height to be $\sim 300^{\prime \prime}$. If we assume the height of the original loops at the flare onset to be $50^{\prime \prime}$ (as there are no STEREO observations of this active region immediately after the flare, we assume this height as a common value for ordinary flares), this yields a radial velocity of $\sim 2 \mathrm{~km} \mathrm{~s}^{-1}$ when averaged over the whole day. This is similar to typical speeds of rising post-flare loops very late in an event (e.g., MacCombie \& Rust 1979; Gallagher et al. 2002), giving further evidence that the NUSTAR source is indeed associated with the flare that occurred a day earlier.

\section{ANALYSIS OF THE HIGH CORONAL SOURCE}

\subsection{Spectral Fitting}

We fitted the NuSTAR count spectrum inside the region of interest from Figure 2 separately for FPMA and FPMB, following the approach of Hannah et al. (2016), using SolarSoft/OSPEX. ${ }^{16}$ The counts were binned with $0.2 \mathrm{keV}$

\footnotetext{
${ }^{16}$ http://hesperia.gsfc.nasa.gov/ssw/packages/spex/doc/
} 

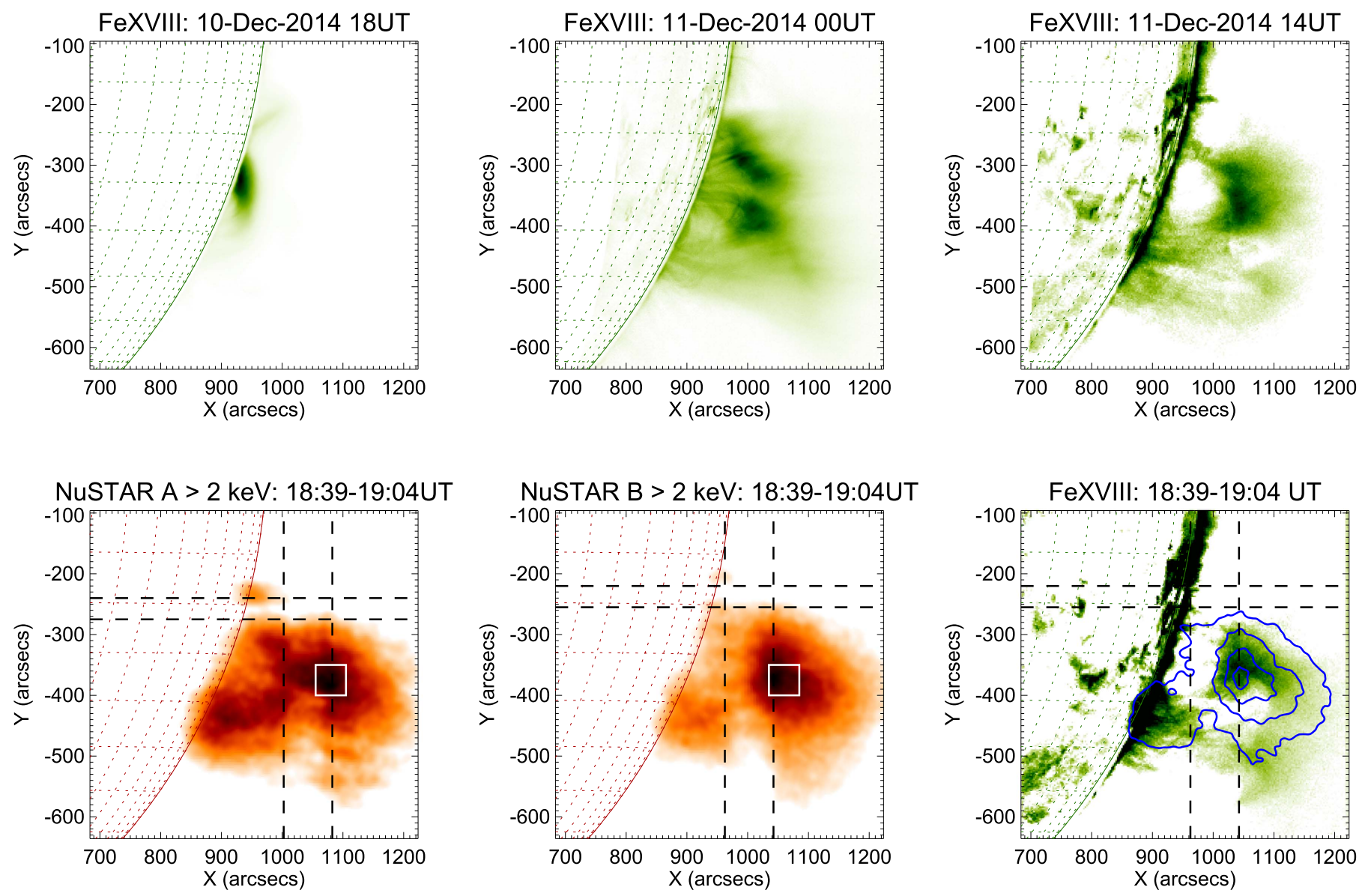

Figure 2. Upper row: Fe XVIII maps of the flare onset (left panel), post-flare loops $6 \mathrm{hr}$ after the flare (middle panel), and the remaining loops $20 \mathrm{hr}$ after the flare (right panel). Bottom row: 25-minute integrated NuSTAR FPMA (left panel) and FPMB (middle panel) and AIA Fe XVIII (right panel) maps; the latter includes the 30\%, $50 \%, 70 \%$, and $90 \%$ NUSTAR contours in blue. Dashed lines denote the area affected by the NuSTAR chip gap during this observation. The white box is the region chosen for the spectral analysis.

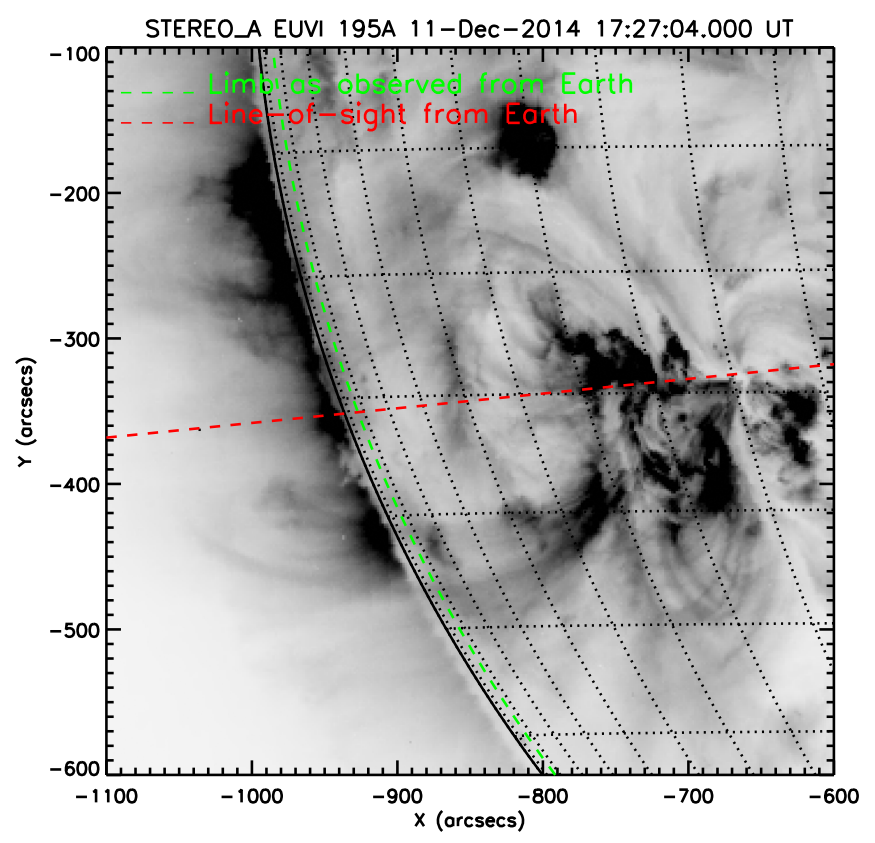

Figure 3. STEREO-A $195 \AA$ image of active region AR 12222 an hour before the NUSTAR observations. The orange line presents the solar limb as viewed from Earth, while the red line is the line of sight from Earth through the NUSTAR source. energy resolution, while the integration time was 25 minutes (full NUSTAR observing time of the active region). As the livetime was around $1 \%$ during the whole observation period, this is roughly equal to $15 \mathrm{~s}$ of exposure at full livetime. In order to investigate the influence of the adopted energy range on the fitted temperature and emission measure, we fitted CHIANTI 7.1 isothermal models (Dere et al. 1997; Landi et al. 2013) to our data for different energy ranges: $2.5-5.2 \mathrm{keV}, 3.0-5.2 \mathrm{keV}$, $3.5-5.2 \mathrm{keV}, 4.0-5.2 \mathrm{keV}$. These fits are presented in Figure 4. The lower limit of $2.5 \mathrm{keV}$ was chosen as the lowest energy for which the calibration is still completely understood and reliable (Grefenstette et al. 2016), while the upper limit of $5.2 \mathrm{keV}$ was chosen as the highest energy with a significant number of counts ( $>3$ counts per bin). Both focal plane modules give consistent results, with temperature $3.8-4.6 \mathrm{MK}$ and emission measure $(0.3-1.8) \times 10^{46} \mathrm{~cm}^{-3}$, depending on the lower limit of the energy range used in the fitting. The temperature gets higher and the emission measure gets lower as we go to higher energies. The 67\% confidence ranges of temperature and emission measure were calculated using the standard Monte Carlo procedure in OSPEX and are given in Table 1, together with the best-fit values. A point to note is that our region of interest is located very close to the gap between the detectors, which leads to fewer counts, especially in later phases of the integration interval. The reason for this is the slow drift of the spacecraft 

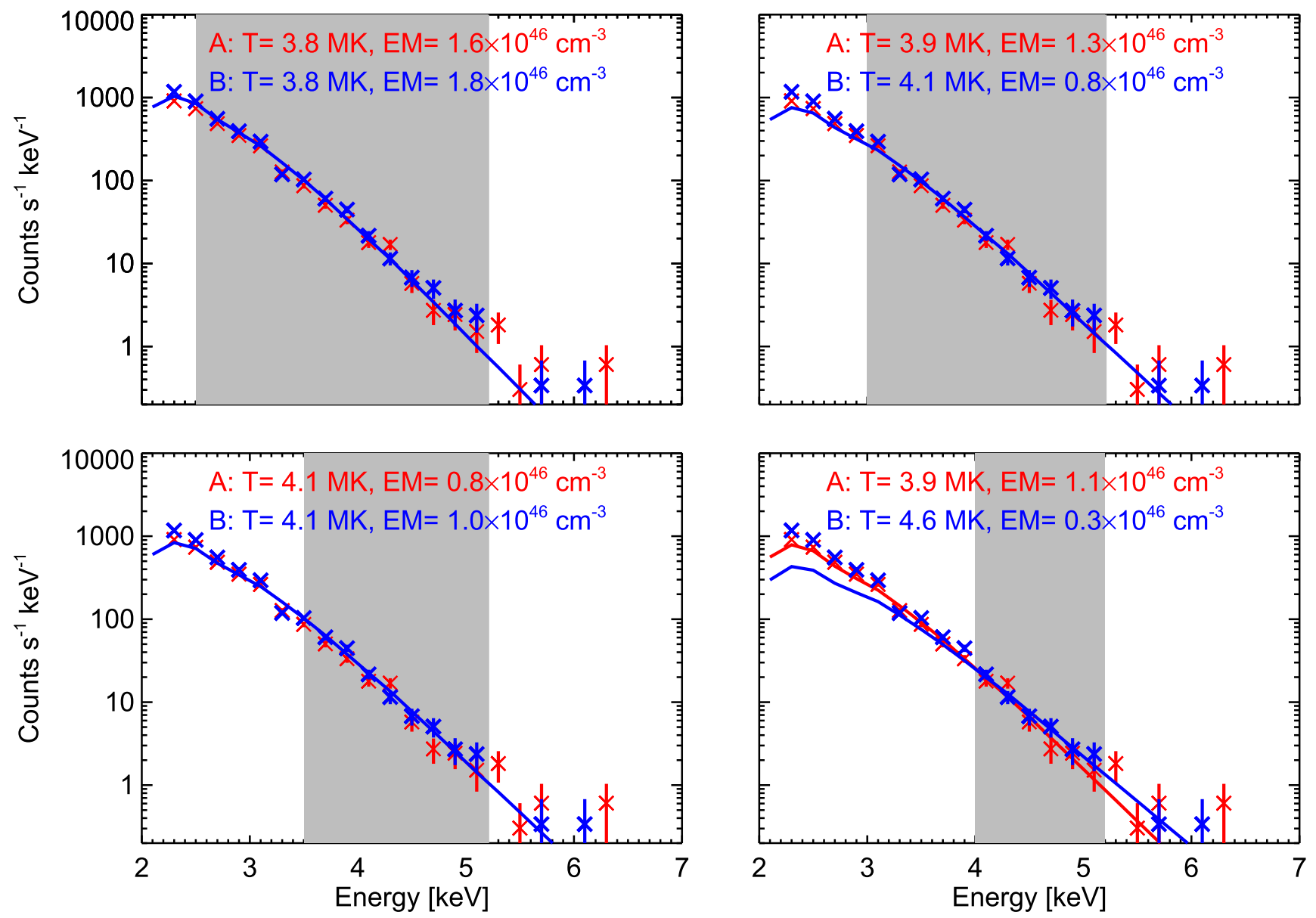

Figure 4. NUSTAR count spectra for FPMA (red) and FPMB (blue) integrated over the whole observation time range (18:39-19:04), together with isothermal fits for different energy ranges: $2.5-5.2 \mathrm{keV}$ (upper left), 3.0-5.2 keV (upper right), 3.5-5.2 keV (lower left), and 4.0-5.2 keV (lower right). Energy ranges for spectral fitting are shown with gray shaded areas. The best-fit values of temperature and emission measure for individual focal plane modules can be found on the top of each graph.

Table 1

Best-fit Values of Temperature and Emission Measure and Their 67\% Confidence Ranges

\begin{tabular}{lrrrr}
\hline \hline \multicolumn{5}{c}{ FPMA } \\
\hline Energy Range (keV) & $2.5-5.2$ & $3.0-5.2$ & $3.5-5.2$ & $4.0-5.2$ \\
\hline$T(\mathrm{MK})$ & $3.77_{-0.04}^{+0.04}$ & $3.86_{-0.09}^{+0.09}$ & $4.05_{-0.16}^{+0.18}$ & $3.94_{-0.44}^{+0.43}$ \\
EM $\left(10^{46} \mathrm{~cm}^{-3}\right)$ & $1.60_{-0.12}^{+0.14}$ & $1.30_{-0.23}^{+0.28}$ & $0.82_{-0.25}^{+0.36}$ & $1.11_{-0.72}^{+3.18}$ \\
\hline \multicolumn{5}{c}{ FPMB } \\
\hline Energy range $(\mathrm{keV})$ & $2.5-5.2$ & $3.0-5.2$ & $3.5^{-5} .2$ & $4.0-5.2$ \\
\hline$T(\mathrm{MK})$ & $3.79_{-0.05}^{+0.04}$ & $4.12_{-0.10}^{+0.10}$ & $4.06_{-0.16}^{+0.16}$ & $4.57_{-0.45}^{+0.62}$ \\
EM $\left(10^{46} \mathrm{~cm}^{-3}\right)$ & $1.75_{-0.12}^{+0.15}$ & $0.84_{-0.15}^{+0.17}$ & $0.99_{-0.28}^{+0.44}$ & $0.30_{-0.20}^{+0.77}$ \\
\hline
\end{tabular}

pointing with time, resulting in covering a part of the region of interest by the gap. The missing counts could lead to an underestimation of the emission measure, but do not change the value of the determined temperature (as it is determined by the slope in the counts spectrum). A single-temperature component is enough to fit the observations, similar to the results of Hannah et al. (2016). We determine the density of the source to be (assuming a volume of $50 \times 50 \times 50^{1 / 3}$ ) in the range of $(2.5-6.0) \times 10^{8} \mathrm{~cm}^{-3}$ (roughly 10-100 times the density of the quiet-Sun corona at this height; see, e.g., Withbroe 1988), suggesting that the density of late-phase loops is significantly higher than that of the quiet-Sun corona.

\subsection{Comparison of NUSTAR to SDO/AIA}

\subsubsection{Comparison to Fe XVIII}

In order to investigate the extent of the agreement between NUSTAR and Fe XVIII sources, we compare the Fe XVIII loci curve with the NUSTAR loci curves in different energy channels. For reference, the results of NUSTAR spectral fitting from the previous section for both focal plane modules are presented in Figure 5, with different symbols for different energy ranges, together with the Fe XVIII and NuSTAR loci curves. The FeXVIII loci curve is extracted from the temperature response functions (Boerner et al. 2014) and the observed fluxes using the following formula:

$$
\mathrm{EM}=\frac{F \cdot S}{R(T)},
$$

where EM is the emission measure $\left[\mathrm{cm}^{-3}\right], F$ is the flux [DN $\mathrm{s}^{-1}$ pixel $\left.^{-1}\right], S$ is the area of the region $\left[\mathrm{cm}^{2}\right]$, and $R(T)$ is the temperature response function of the FeXVIII line $\left[\mathrm{DNcm}^{5} \mathrm{~s}^{-1}\right.$ pixel $\left.^{-1}\right]$. The NUSTAR loci curves are extracted in a similar way from the NUSTAR temperature response function, determined by folding the generated photon spectra for different temperatures through the NUSTAR response matrix. The good agreement of our results is best seen in the inset of Figure 5, where we plot the loci curves and the determined EM- $T$ pairs on a linear scale. The intersection of 


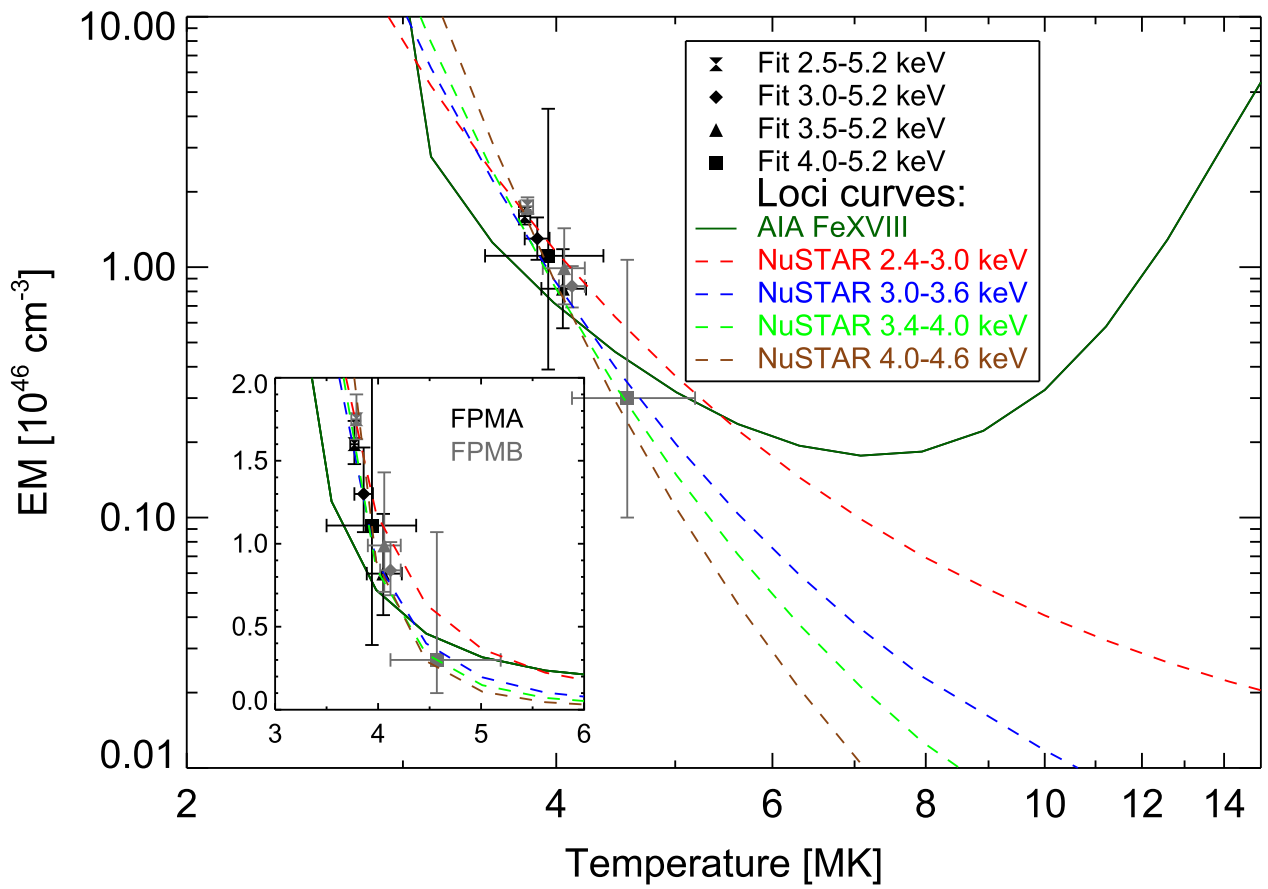

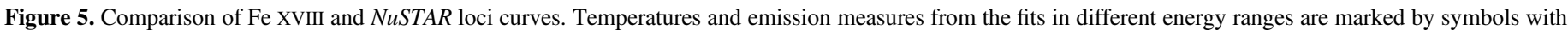

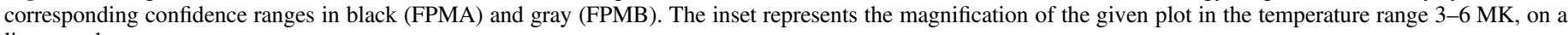
linear scale.

the Fe XVIII loci curve with the NUSTAR loci curves in the temperature range $4.0-4.3 \mathrm{MK}$ is consistent with the EM-T pairs shown in Figure 4, except for the fit including the lowest energies. A part of these low-energy counts might originate from cooler post-flare loops, which will also be discussed in more detail in the next sections.

\subsubsection{Comparison to Other AIA Channels}

It is also possible to investigate the results of NuSTAR fitting to other AIA channels by calculating the expected count rates in different AIA channels from the source with the emission measure and temperature as given by NuSTAR, and compare them with the observed fluxes in AIA maps. The difficulty of this comparison is that the fraction of the cold background emission (in the temperature range below $\sim 3 \mathrm{MK}$ ) in these channels is unknown and non-removable. This is not an issue for the derived Fe XVIII channel, which is not sensitive to this cooler plasma. The expected AIA fluxes are calculated by inverting Equation (3). This is a NuSTAR-predicted AIA flux coming from the NUSTAR source alone, without any additional contribution from the cooler plasma. The comparison between NuSTAR-predicted and observed fluxes is presented in Figure 6. The circles are the predicted fluxes for NuSTAR spectral fitting in the range $2.5-5.2 \mathrm{keV}$, and the stars are for $4.0-5.2 \mathrm{keV}$. We use the fitted values of FPMB in both ranges, as they represent the two extreme $T$-EM fits. The full and dashed lines represent $1 \%, 5 \%, 10 \%, 50 \%$, and $100 \%$ ratios of NuSTARpredicted and observed fluxes in different AIA channels. The area where the predicted AIA flux from the NUSTAR source is larger than the total observed flux is shown with the red lines. If the NuSTAR -predicted flux for a given AIA channel is close to the observed flux (e.g., region between 50\% and $100 \%$ lines in the plot), the emission in that AIA channel is dominated by the same plasma that NuSTAR observes. Unsurprisingly, this is best

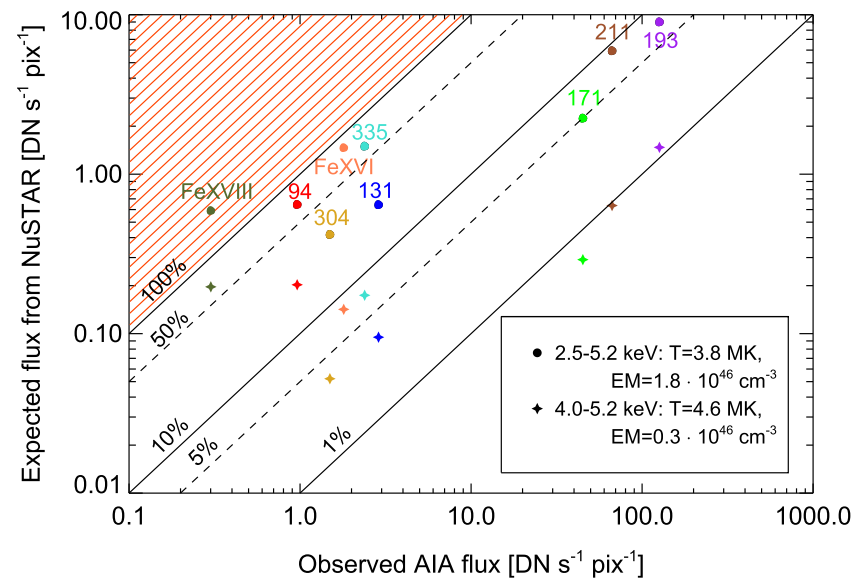

Figure 6. Comparison of expected and observed fluxes for seven AIA channels and the derived Fe XVI and Fe XVIII channels, for the two extreme pairs of temperature and emission measure (values given in the legend) from fits in Figure 4 . The diagonal lines denote $1 \%, 5 \%, 10 \%, 50 \%$, and $100 \%$ ratios of the expected AIA fluxes from the NUSTAR source and the observed AIA fluxes. The red lines denote the (forbidden) area where the predicted AIA flux from the NUSTAR source is larger than the total observed AIA flux.

achieved for the $94 \AA$ channel and, consequently, the Fe XVIII channel. For the first $T-\mathrm{EM}$ fit, the NuSTAR-predicted flux for the Fe XVIII channel is greater than the observed flux. This result indicates that a single-temperature fit is not enough to fit the observations at the lowest energies, as some of the lowenergy counts are produced by a lower-temperature plasma. The ratio for the Fe XVIII channel for the fit at higher energies (second $T-$ EM fit) lies in the range between $50 \%$ and $100 \%$, while the $335 \AA$ channel and its derived Fe XVI channel have ratios in the range of $5 \%-10 \%$. These results are in agreement with the fact that the Fe XVIII source showed the same spatial features as the NUSTAR source, while we were not able to detect the Fe XVI source. Cooler lines at 171, 211, and $193 \AA$ 
have ratios of NUSTAR-predicted fluxes to the observed fluxes at a percent level, which is expected as these lines are sensitive to plasma cooler than NUSTAR can observe.

\subsection{Comparison of NUSTAR to FOXSI}

The FOXSI (Krucker et al. 2014) sounding rocket also uses direct focusing HXR optics, but is optimized especially for solar purposes. FOXSI has about one-fifth of NUSTAR's effective area, with a higher spatial resolution (FWHM of 9 "). The main difference for solar observations between the two telescopes is the different low-energy threshold. While NUSTAR detects photons down to $\sim 2 \mathrm{keV}$, the FOXSI entrance window intentionally blocks the large number of low-energy photons, giving a typical peak in the count spectrum around $5 \mathrm{keV}$. The entrance window largely reduces the number of incoming photons, keeping the livetime high for the faint, higher-energy components. For example, a 25-minute observation by NUSTAR at $1 \%$ livetime and five times the effective area is equal to a FOXSI observation of $75 \mathrm{~s}$ at full livetime. However, this also means that FOXSI is not sensitive to lowtemperature plasmas that are best seen below $4 \mathrm{keV}$.

The FOXSI-2 rocket flew for a 6.5-minute observation interval during the NUSTAR solar pointing discussed here. FOXSI-2 targeted AR 12222 for $35.2 \mathrm{~s}$, though 12 minutes after the NUSTAR observation finished. As the NuSTAR/AIA source has a slow time variation, the time difference between the observations is of minor importance, at least for the order-ofmagnitude estimate discussed here. Using the temperature and emission measure derived from NUSTAR $(T=3.8 \mathrm{MK}$ and $\mathrm{EM}=1.7 \times 10^{46} \mathrm{~cm}^{-3}$ ), the expected FOXSI count rate is $\sim 1.6$ counts for FOXSI-2's most sensitive optics/detector pair D6. This value is computed above $5 \mathrm{keV}$ and with the integration time of $35.2 \mathrm{~s}$ (integrating during the whole observation period). In total, 4 counts were observed by D6. This is a reasonable value given that the estimated nonsolar background flux is 1.8 counts, while the expected count rate due to ghost-rays from sources outside of the FOV is unknown. Given the small number statistics and the uncertainty of the ghost-ray background, the observed FOXSI-2 measurement is consistent with the values expected for the plasma observed with NUSTAR, but does not provide any further diagnostics for this event.

\section{DICUSSION AND CONCLUSIONS}

In this paper, we have presented the first observations of the EUV late phase of a solar flare in X-rays with NuSTAR. NUSTAR has provided a unique opportunity to perform spectroscopy on X-rays from a coronal source a full day after the flare onset. With knowledge of the location of this faint source from NuSTAR, we were also able to find it in Fe XVIII by eliminating the lower-temperature response of the AIA $94 \mathrm{~A}$ channel and integrating for 25 minutes (adding together 125 maps to obtain a higher signal-to-noise ratio). Here, NuSTAR played a crucial role in providing the information needed for extracting the very faint signal that was far from evident in the $94 \AA$ maps.

The fact that the post-flare loops have been observed so late in the flare evolution points to continuing energy input in the later phases of the solar flare evolution. To quantify this statement, we estimate the cooling times of subsequent postflare loops and compare them to the flare duration. We follow the approach of Cargill et al. (1995), with the following formula for the cooling time of post-flare loops:

$$
\tau_{\text {cool }}=2.35 \cdot 10^{-2} \cdot L^{5 / 6} \cdot n_{e}^{-1 / 6} \cdot T_{e}^{-1 / 6},
$$

where $\tau_{\text {cool }}[\mathrm{s}]$ is the cooling time (the time needed for postflare loops to cool down to $\sim 10^{5} \mathrm{~K}$ ) and $L[\mathrm{~cm}], n_{e}\left[\mathrm{~cm}^{-3}\right]$, and $T_{e}[\mathrm{~K}]$ are loop length, density, and temperature at the start time, respectively. The temperature estimate of the original post-flare loops from the GOES observations is $10.5 \mathrm{MK}$, while the emission measure is $5 \times 10^{48} \mathrm{~cm}^{-3}$. Even though the above estimates might only be a rough approximation because of the high occultation of the flare, we are anyway making only an approximate calculation of the cooling time. By assuming the length of the original post-flare loops to be $\sim 50^{\prime \prime}$, we estimate the density to be $9 \times 10^{9} \mathrm{~cm}^{-3}$. This gives us a cooling time of $\sim 1 \mathrm{hr}$, indicating that the original post-flare loops are long gone at the time of NUSTAR observations and that the additional heating took place during the evolution of the post-flare system. The most probable explanation is the previously mentioned scenario of subsequent magnetic reconnections, resulting in reconnected loops being produced higher and higher in the corona.

The above results are in agreement with original Skylab and Solar Maximum Mission results and the recent observations of a large post-flare loop system between 2014 October 14 and 16 by West \& Seaton (2015). They conclude that the giant latephase arches are similar in structure to the ordinary post-flare loops and formed by magnetic reconnection. Their reasoning follows the work of Forbes \& Lin (2000), in which it is pointed out that the reconnection rate may not depend only on the magnetic field (in which case it would decrease with height), but possibly also on the local Alfvén speed, which is proportional to $B / \sqrt{\rho}$, where $B$ is the magnetic field strength and $\rho$ the density. So, if the density decreases sufficiently fast, the reconnection rate could remain constant out to $0.5 R_{\odot}$, despite the decreasing magnetic field strength, and thus produce the giant post-flare loops analyzed by West \& Seaton (2015) or in this study.

From NUSTAR and GOES data, it is possible to estimate the additional energy input needed to form the subsequent, rising post-flare loops. The total thermal energy of the loop system is proportional to the density, temperature, and volume (e.g., Hannah et al. 2008):

$$
E_{\text {th }}=3 N k T=3 k \cdot n V T,
$$

where $k$ is the Boltzmann constant. We have obtained all the above parameters for the original flare loops from GOES and for the post-flare loops a day after from NUSTAR. We estimate that the thermal energy content in NUSTAR loops is 5\% of the thermal energy content of the original flare loops, indicating that there is still significant energy release even a full day after the flare onset. Next, by assuming linearity in the change of density, loop length, and temperature over time (for simplicity), it is possible to calculate the change in cooling times of all the post-flare loops formed in between. Although the above assumption might not be accurate for all (or any) of the parameters, we are only interested in calculating an order-ofmagnitude estimate here. The other assumption we use is that new loop systems are only produced when the old ones vanish. This assumption is in principle not valid as new systems are 
produced while the old ones persist, but it gives us an approximate lower limit on the total thermal energy content in all the loop systems. The sequence is as follows: original postflare loops vanish after $\sim 1 \mathrm{hr}$, and during this time, density, temperature, and volume change as well, and a new loop system with a different cooling time is produced. We calculate that this sequence repeats about 12 times during the $24 \mathrm{hr}$ between the flare onset and NuSTAR observations, with the total energy content in those 12 cycles of reconnection and cooling estimated at a factor of $\sim 13$ larger than the one released during the impulsive phase of the flare only.

Previous estimates of the additional energy input during the decay phase of solar flares were derived using radiative losses at specific wavelength ranges. Woods et al. (2011) calculate the total radiated energy in the EUV band during the late phase to be between 0.4 and 3.7 times the flare energy in the X-rays during the peak. Emslie et al. (2012) conclude in their statistical study of 38 solar flares that, on average, the total energy radiated from hot SXR-emitting plasma exceeds the peak thermal energy content by a factor of $\sim 3$. It is important to note that the above studies used non-overlapping wavelength ranges, thus missing the contribution to total energy content from the wavelength range of the other study (and the rest of the wavelength spectrum). Our results for a single event are consistent with these statistical studies, especially as we compare our value with statistical averages that miss significant energy contributions.

In summary, all results indicate that the impulsive energy release is only a fraction of the energy release in the late phase of the flare evolution, at least for events with clearly observable late-phase emission. This statement calls for re-examining the approach of using just the peak energy content or the nonthermal emission during the impulsive phase of the flare as the estimate of the total energy content of the flare. In order to assess this in more detail, a statistical study of similar events should be carried out. However, NUSTAR is not a solardedicated observatory, and therefore the observations are few and sporadic, making statistical studies difficult. Additionally, it is most likely that faint signals such as presented in this study can only be observed when the flare (and the active region) is occulted or at least over the limb, as the emission from these kinds of coronal sources on the disk would likely be masked by the much stronger emission of the active region beneath. Nevertheless, a statistical search for SDO/AIA Fe XVIII sources in above-the-limb flares could give us new insights about the influence of the long-lasting decay phase on flare energetics.

This work made use of data from the NUSTAR mission, a project led by the California Institute of Technology, managed by the Jet Propulsion Laboratory, and funded by NASA. We thank the NuSTAR Operations, Software and Calibration teams for support with the execution and analysis of these observations. This research made use of the NuSTAR Data Analysis
Software (NuSTARDAS), jointly developed by the ASI Science Data Center (ASDC, Italy) and the California Institute of Technology (USA). M.K. and S.K. acknowledge funding from the Swiss National Science Foundation (200021-140308). Funding for this work was also provided under NASA grants NNX12AJ36G and NNX14AG07G. A.J.M.'s participation was supported by NASA Earth and Space Science Fellowship award NNX13AM41H. I.G.H. is supported by a Royal Society University Research Fellowship. P.J.W. is supported by an EPSRC-Royal Society fellowship engagement grant. FOXSI was funded by NASA LCAS grant NNX11AB75G. We would also like to thank the anonymous referee for the helpful comments.

\section{REFERENCES}

Boerner, P. F., Testa, P., Warren, H., Weber, M. A., \& Schrijver, C. J. 2014, SoPh, 289, 2377

Cargill, P. J., Mariska, J. T., \& Antiochos, S. K. 1995, ApJ, 439, 1034

Del Zanna, G. 2013, A\&A, 558, A73

Dere, K. P., Landi, E., Mason, H. E., Monsignori Fossi, B. C., \& Young, P. R. 1997, A\&AS, 125, 149

Emslie, A. G., Dennis, B. R., Shih, A. Y., et al. 2012, ApJ, 759, 71

Fárník, F., Švestka, Z., Hudson, H. S., \& Uchida, Y. 1996, SoPh, 168, 331

Forbes, T. G., \& Lin, J. 2000, JASTP, 62, 1499

Gallagher, P. T., Dennis, B. R., Krucker, S., Schwartz, R. A., \& Tolbert, A. K. 2002, SoPh, 210, 341

Grefenstette, B. W., Glesener, L., Krucker, S., et al. 2016, ApJ, 826, 20

Hannah, I. G., Christe, S., Krucker, S., et al. 2008, ApJ, 677, 704

Hannah, I. G., Grefenstette, B. W., Smith, D. M., et al. 2016, ApJL, 820, L14

Harrison, F. A., Craig, W. W., Christensen, F. E., et al. 2013, ApJ, 770, 103

Howard, T. A., \& Harrison, R. A. 2004, SoPh, 219, 315

Hudson, H. S., Lemen, J. R., St., Cyr, O. C., Sterling, A. C., \& Webb, D. F. 1998, GeoRL, 25, 2481

Kopp, R. A., \& Poletto, G. 1984, SoPh, 93, 351

Krucker, S., Christe, S., Glesener, L., et al. 2014, ApJL, 793, L32

Landi, E., Young, P. R., Dere, K. P., Del Zanna, G., \& Mason, H. E. 2013, ApJ, 763, 86

Lin, R. P., Dennis, B. R., Hurford, G. J., et al. 2002, SoPh, 210, 3

Liu, K., Zhang, J., Wang, Y., \& Cheng, X. 2013, ApJ, 768, 150

MacCombie, W. J., \& Rust, D. M. 1979, SoPh, 61, 69

Madsen, K. K., Harrison, F. A., Markwardt, C. B., et al. 2015, ApJS, 220, 8

McIntosh, S. W., Leamon, R. J., Davey, A. R., \& Wills-Davey, M. J. 2007, ApJ, 660, 1653

Nitta, N. V., Aschwanden, M. J., Boerner, P. F., et al. 2013, SoPh, 288, 241

Parenti, S., Reale, F., \& Reeves, K. K. 2010, A\&A, 517, A41

Reale, F., Guarrasi, M., Testa, P., et al. 2011, ApJL, 736, L16

Rust, D. M., \& Hildner, E. 1976, SoPh, 48, 381

Stewart, R. T., McCabe, M. K., Koomen, M. J., Hansen, R. T., \& Dulk, G. A. 1974, SoPh, 36, 203

Švestka, Z. 1984, SoPh, 94, 171

Švestka, Z., Dennis, B. R., Woodgate, B. E., et al. 1982, SoPh, 80, 143

Švestka, Z., Fárník, F., Hudson, H. S., et al. 1995, SoPh, 161, 331

Testa, P., \& Reale, F. 2012, ApJL, 750, L10

Warren, H. P., Winebarger, A. R., \& Brooks, D. H. 2012, ApJ, 759, 141

West, M. J., \& Seaton, D. B. 2015, ApJL, 801, L6

Withbroe, G. L. 1988, ApJ, 325, 442

Woods, T. N. 2014, SoPh, 289, 3391

Woods, T. N., Hock, R., Eparvier, F., et al. 2011, ApJ, 739, 59

Zarro, D. M., Sterling, A. C., Thompson, B. J., Hudson, H. S., \& Nitta, N. 1999, ApJL, 520, L139 